\title{
Nuevos ambientes de aprendizaje observados desde un enfoque sistémico a los docentes de la Universidad Católica de Cuenca - Carrera Sicología Clínica
}

\section{New learning environments observed from a systemic approach to teachers at the Catholic University of Cuenca - Career Clinical Psychology}

\author{
Alvarez Guzhñay, Pedro César ${ }^{1 *}$, Alvarado Maldonado, Hugo Patricio ${ }^{1}$ y Cabrera Vintimilla, Henry Paul $^{1}$ \\ ${ }^{1}$ Universidad Católica de Cuenca, Ecuador \\ *palvarez@ucacue.edu.ec
}

\begin{abstract}
Resumen
Desde la perspectiva de la Universidad Católica de Cuenca como una institución de Educación Superior, se determinó la necesidad de conocer, cómo los docentes de la Carrera de Sicología Clínica, aplican los nuevos ambientes de aprendizaje de tipo virtual en el proceso de enseñanza-aprendizaje; así mismo, identificar las actividades y los recursos que se encuentran plasmadas en su micro currículo (syllabus) y que reposan en la plataforma virtual de enseñanza aprendizaje (Moodle) de la Universidad Católica de Cuenca. Para ello, se realizó un análisis partiendo desde el enfoque sistémico, el cual nos permitió observar, identificar y relacionar estas actividades con el proceso de enseñanza-aprendizaje que día tras día llevan los docentes hacia sus estudiantes. En este marco, el presente artículo aporta una propuesta para el monitoreo y la correcta aplicación de estos recursos y actividades que se encuentran preestablecidos en una plataforma de tipo virtual y su impacto en el proceso de acreditación en función al modelo de evaluación de carreras emitido por el Consejo de Evaluación, Acreditación y Aseguramiento de la Calidad de la Educación Superior (CEAACES), llegando a obtener resultados medianamente satisfactorios.
\end{abstract}

Palabras clave: Nuevos ambientes de aprendizaje, syllabus, Moodle, CEEACES.

\begin{abstract}
From the perspective of the Universidad Católica de Cuenca as an institution of Higher Education, it was determined the need to know, how the teachers of the Career of Clinical Psychology, apply new learning environments of virtual type in the teaching-learning process; as well as to identify the activities and resources that are embodied in their syllabus and that are based on the virtual learning platform (Moodle) of the Catholic University of Cuenca. For this, an analysis was made starting from the systemic approach, which allowed us to observe, identify and relate these activities to the teachinglearning process that day after day teachers bring to their students. In this context, this article provides a proposal for the monitoring and correct application of these resources and activities that are preset in a virtual type platform and its impact on the accreditation process according to the model of career evaluation issued by the Council for Evaluation, Accreditation and Quality Assurance of Higher Education (CEAACES), reaching satisfactory results.
\end{abstract}

Key words: New learning environments, syllabus, Moodle, CEEACES..

\section{Introducción}

Las Tecnologías de la Información y Comunicación, son un elemento muy importante en la interacción entre individuos y sociedades, estos nuevos espacios virtuales conllevan rasgos diversos, que generan la necesidad de un análisis entorno a sus características.

En la sociedad actual los espacios educativos se encuentran inmersos en grandes cambios; surgen nuevos ámbitos informáticos que se encuentran orientados a la educación y es aquí donde estos nuevos escenarios requieren de una reflexión crítica de cómo los ambientes de aprendizaje de tipo virtual se encuentran aplicados para el proceso de enseñanza - aprendizaje, observados desde un enfoque sistémico.

Hay que observar que las Tecnologías Informáticas pueden llegar a ser utilizadas como un medio o un recurso didáctico, es decir, pueden presentar soluciones o ayudas pedagógicas, pero no enfocándose en que la tecnología puede hacer todo el trabajo en el proceso de enseñanza aprendizaje. 
Para poder identificar cómo las tecnologías de la información y la comunicación y dentro de ellas los nuevos ambientes de aprendizaje de tipo virtual. El enfoque sistémico posibilitará, el análisis, la reflexión y el estudio en forma de un sistema, generando las relaciones entre docentes, estudiantes con los nuevos ambientes virtuales en el proceso de enseñanza.

\section{Marco téorico}

Daniel Raichvarg en 1994 define que la palabra ambiente data desde 1921 y fue expuesta por un grupo de geógrafos que consideraban como la interacción del hombre con el entorno que lo rodea (Duarte, 2003, p. 3). Es decir, una interacción hombre-naturaleza. Según la Real Academia de la Lengua Española, la palabra Ambiente hace referencia a lo que rodea a todo ser humano o a alguien que se encuentre como un elemento de un entorno.

Por otro lado, Sauvé en 1994, fundamenta que la noción ambiente se ha derivado a otros ámbitos en la vida del hombre y pueden llegar a ser: la cultura y la educación como aspectos principales. La observación y aplicabilidad de un ambiente en la educación han sido de gran importancia ya que han permitido identificar seis concepciones básicas que se detallan a continuación:

1) Ambiente como problema

2) Ambiente como recurso

3) Ambiente como naturaleza

4) Ambiente como biósfera

5) Ambiente como medio de vida $y$

6) Ambiente comunitario.

Cada una de estas concepciones ayudan a definir su relación con la educación, permitiendo identificar que la palabra ambiente resulta ser una realidad compleja, es decir, solo puede ser abordada desde una pluralidad de varios saberes.

Una vez realizado un breve recorrido sobre algunos conceptos en cuanto a la definición de ambiente, se hace necesario una conceptualización teórica la cual permitirá definir más claramente el objeto de estudio de este artículo.

\section{Ambiente Educativo}

Un ambiente educativo es un espacio organizado y estructurado con el fin de facilitar el proceso de enseñanza dentro del aula de clases, también es conocido, como la construcción diaria del conocimiento. Otra definición a mencionar es que un ambiente educativo es un espacio y tiempo donde los participantes (Docentes - Estudiantes), desarrollan capacidades, competencias y valores.

Otra de las nociones de ambiente educativo remite al escenario donde existen y se desarrollan condiciones favorables de aprendizaje. Un espacio y un tiempo en movimiento, donde los participantes desarrollan capacidades, competencias, habilidades y valores (A.C. CEP Parras 1997: 15-18)

Es importante mencionar que un ambiente educativo no se limita a condiciones materiales de un currículo, es decir, no se encuentra estático, por lo contrario, incorpora dinámicas que constituyen procesos educativos que involucran acciones, vivencias y experiencias de cada uno de sus participantes fomentando una mejora educativa en el proceso de enseñanza aplicado a cualquier nivel educativo ya sea inicial, primario, secundario o superior (ECURED, 2001).

\subsection{Nuevos Ambientes de Aprendizaje (Virtuales) \\ Contexto de Cambios}

Se puede mencionar que el sistema educativo y el uso de las tecnologías son consecuencias de la revolución industrial, donde se hace referencia al hecho de que la sociedad necesitaba buscar nuevas condiciones para tratar de mejor manera al ser humano, es por ello que permitió el acceso a instituciones de educación con el fin de dar soluciones a las problemáticas que se presentan en el mundo, al incluirla con la tecnología, esta ha permitido cambiar el modelo educativo, enfocándose en nuevos ambientes para un proceso de enseñanza.

El uso de las tecnologías en la educación, plantea muchos desafíos hoy en día, ya que al crecer rápidamente y al ser de vital importancia para la población, contribuye a un vertiginoso cambio en este sector.

Al igual que la llegada de la Primera Revolución Industrial y la que trajo a la sociedad grandes cambios, el uso de las tecnologías en el proceso educativo ha permitido dar un giro de 360 grados, el principal impacto en el aula reside que los estudiantes mantienen un aprendizaje informal, se trata fundamentalmente en la utilización y aplicación de redes formadas por enlaces electrónicos facilitando la adquisición de información y construcción del conocimiento, en esencia este aprendizaje abarca el uso de recursos que se encuentran disponibles en la red de redes (Internet), para procesos de aprendizaje, es decir el estudiante genera un aprendizaje autónomo.

Los escenarios donde el estudiante puede tener acceso para su proceso de enseñanza son:

1) El hogar

2) El puesto de trabajo

3) La escuela

Las perspectivas que ofrecen las Tecnologías de la Información y Comunicación forman un sistema con el fin de enriquecer y mejorar la calidad de la educación, manteniendo la relación informal, docente-estudiante.

\subsection{La Web 2.0}

El término Web 2.0 se le atribuye a Tim O’Reilly y Dale Dougherty en 2004, los cuales lo nombraron durante el transcurso de una sesión de brainstorming, estableciéndolo como "una segunda generación en la historia de la web basada en comunidades de usuarios y una gama especial de servicios y aplicaciones de internet que se modifica gracias a la participación social” (Palomo, R.; Ruiz, J.; Sánchez, J., 2008, p. 13). Es decir, el uso de la misma conlleva una amplia gama de posibilidades en el ámbito educativo, donde como objetivo principal es la de permitir la participación 
social de un estudiante o un grupo de estudiantes de manera real, manteniendo la filosofía del docente como mediador y del estudiante como la persona capaz de construir su propio conocimiento, a cualquier hora y en cualquier lugar sin importar la distancia, solo con mantener una conexión a internet.

\subsection{Recursos Pedagógicos}

La amplia gama de recursos que oferta la Web permite a docente implementarlos para el proceso de enseñanzaaprendizaje, para ello se tomaran como ejemplo los más importantes:

1) $B \log s$

2) Wikis

3) Podcast

4) Redes sociales

5) Plataformas de videos

6) Plataformas virtuales, entre otros más.

Estas herramientas permiten crear al docente una metodología distinta a la hora de enseñar.

\section{Metodología}

Para la elaboración de este artículo, se procedió a utilizar el método bibliográfico, investigando temas de interés en lo referente a los nuevos ambientes de tipo virtual, así también, para el análisis de campo, se tomó como universo y muestra a todos 32 los docentes entre hombres y mujeres que trabajan en la Carrera de Sicología Clínica de la Universidad Católica de Cuenca.

Posterior a ello, se procedió a realizar en análisis sistémico, para ello es necesario conocer cómo se encuentra conformado, esta información se detalla a continuación.

\subsection{Enfoque Sistémico}

Llamado también el enfoque de sistema, permite abordar la relación de objetos y fenómenos como parte de un todo (Sistema), donde se hace referencia a que un sistema no es la suma de elementos, sino es la observación de cómo interactúan, produciendo nuevas características que permiten determinar su comportamiento.

El enfoque sistémico dentro del proceso enseñanza aprendizaje, mantiene una relación e interacción entre docentes y estudiantes, en los cuales como objetivo principal se destaca el objetivo de enseñar, pero para enseñar se debe de cumplir ciertos parámetros establecidos por una Institución de Educación, tomaré como ejemplo la Educación Superior o educación a nivel Universitario en el país del Ecuador, esto en un nivel de supra-sistema, y donde el documento por llamarle así y que permite ser intermediario para el proceso de enseñanza-aprendizaje a nivel docenteestudiante es el Syllabus o silabo, planificación micro curricular el cual y como ejemplo de esta investigación es el sistema a tratar; el mismo debe cumplir requisitos tales como:
1) Objetivo de la importancia de la materia en la planificación curricular.

2) Contenido de o temas de estudio.

3) Método de enseñanza.

4) Ámbitos Educativos (Virtuales, no Virtuales).

5) Evaluaciones y formas de evaluar.

El objetivo principal de esta planificación, es la de integrar nuevos conocimientos en el proceso de enseñanza-aprendizaje. (figura 1)

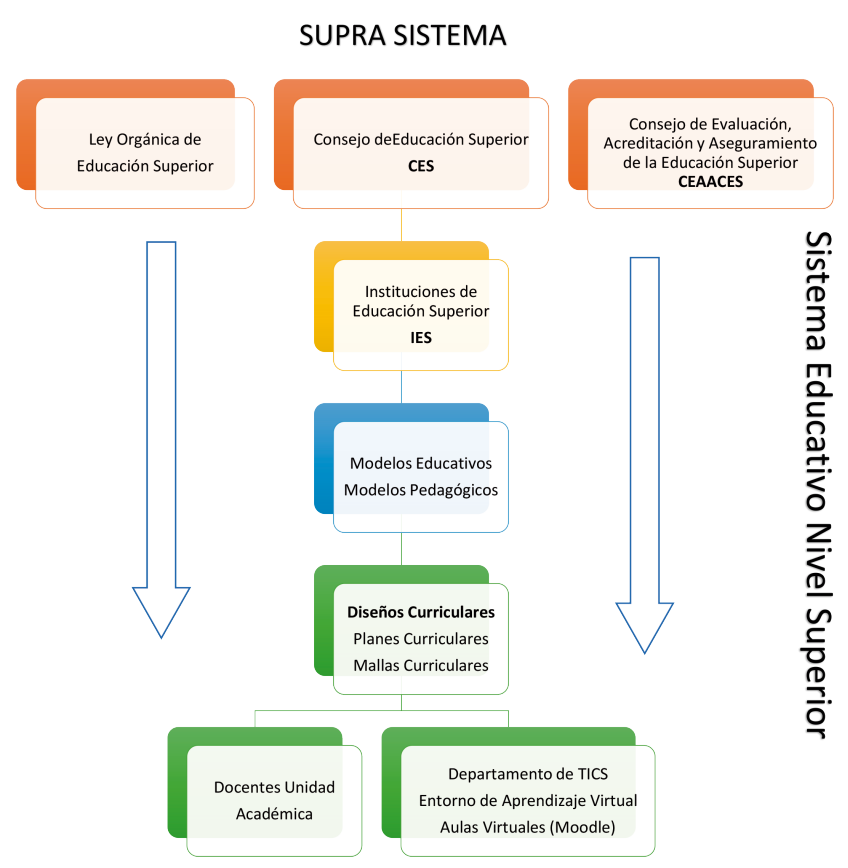

Figura 1. Supra - Sistema - Nivel Educativo

Es por eso que este sistema como se puede observar en la Figura 1, como entradas tiene los reglamentos establecidos por el Consejo de Educación Superior (CES), Consejo de Evaluación, Acreditación, y Aseguramiento de la Calidad de Educación Superior (CEAACES) y a su vez el Reglamento de Régimen Académico aprobado con resolución RPC-So-45-No.535-2014 desarrollado el 17 de diciembre de 2014, donde como uno de sus objetivos se encuentra el uso de ambientes o plataformas virtuales en el proceso de enseñanza - aprendizaje. Relacionado como un sistema de la siguiente manera: 


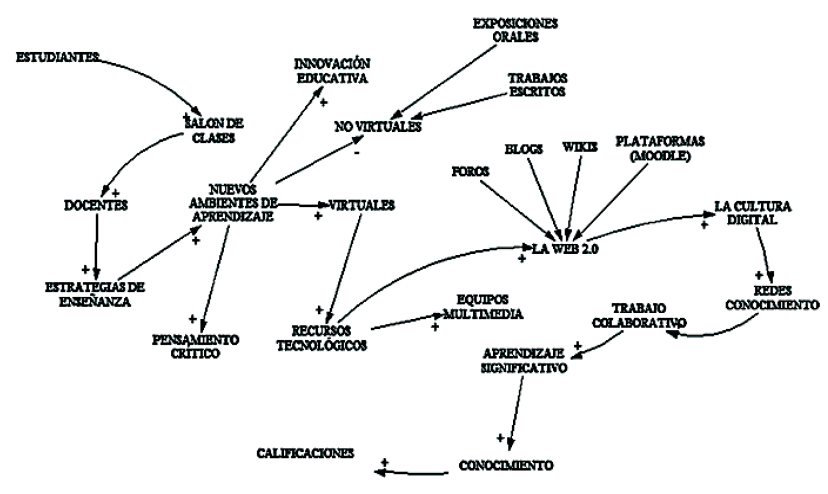

Figura 2. Nuevos ambientes de aprendizaje en la docencia

Como se puede apreciar en la figura 2, esta resulta ser la estructura de los nuevos ambientes de aprendizaje de tipo virtual al aplicar la Web 2.0 dentro de la planificación micro curricular. Se tomo como referencia para la elaboración de este articulo a los 32 docentes que laboran dentro de la Universidad Católica Sicología Clínica, quienes manifestaron que incorporan dentro de la plataforma el trabajo autónomo y colaborativo, ayudando a fomentar la creatividad y la cultura digital hacia sus estudiantes.

Analizando la plataforma virtual Moodle de la Universidad Católica de Cuenca; podemos mencionar que dentro de la misma, esta se encuentra dividida en dos grupos importantes como son: las actividades y los recursos; dentro de las actividades podemos encontrar 13 aplicaciones tales como: Bases de datos, chats, consultas, cuestionarios, encuestas, foros, glosarios, herramientas externas, lecciones, wikis (Web 2.0), solo por citar las más importantes, y a su vez seis recursos como: archivos, carpetas, etiquetas, libros, páginas y direcciones URL. Que buscan lograr y como se citó en párrafos anteriores la construcción del conocimiento y la calidad de la educación.
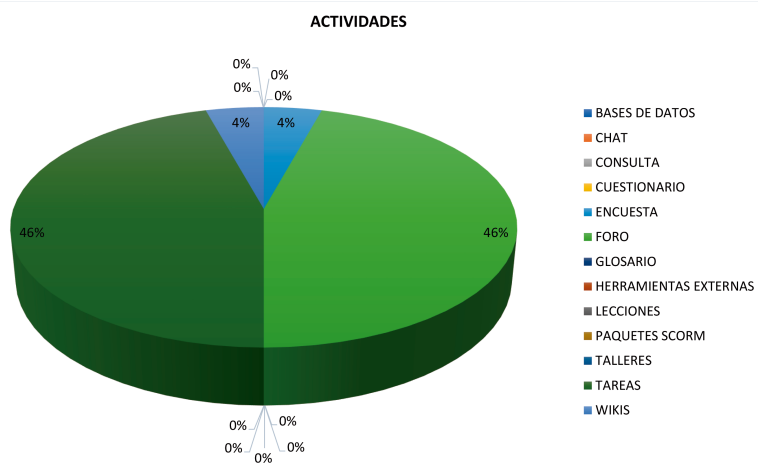

Figura 3. Actividades que utilizan los docentes

En la figura 3, y continuando con el análisis sistémico, se puede apreciar que los 32 docentes de la Carrera de Sicología Clínica, llegan a utilizar cuatro de las 13 activida- des de la plataforma virtual Moodle y entre ellas tenemos: Encuestas, Foros, Tareas y Wikis.

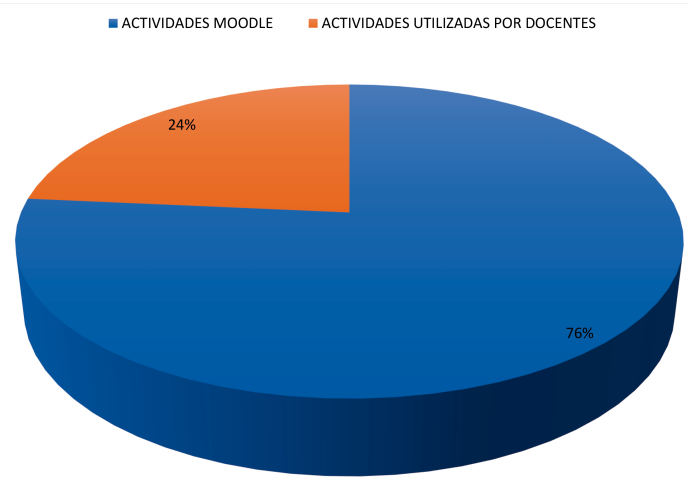

Figura 4. Porcentaje de actividades utilizadas en aula virtual

En la figura 4 hace referencia que las 4 actividades equivalen solo el $24 \%$ de un total de $100 \%$, dejando a un lado otras que son de suma importancia para el proceso de docencia.

Continuando con el análisis, se procedió a verificar que recursos utilizan los docentes de la Carrera de Sicología Clínica en el proceso de enseñanza y se detalla en el gráfico a continuación:

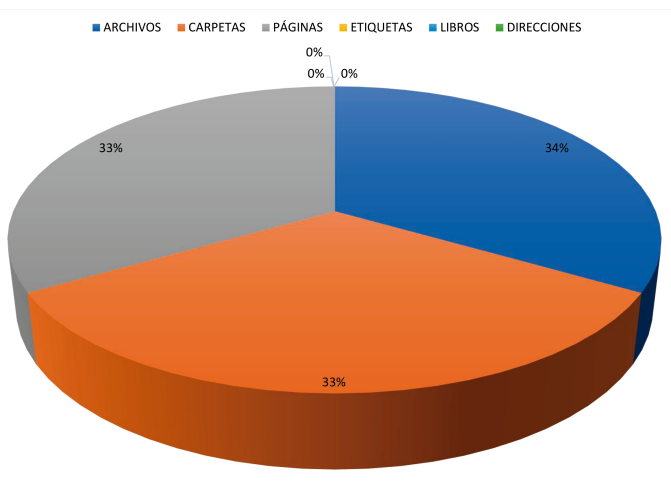

Figura 5. Número de recursos utilizados por los docentes

En la figura 5; de seis recursos que otorga la plataforma, los docentes de la Carrera de Sicología Clínica utilizan solamente tres. 


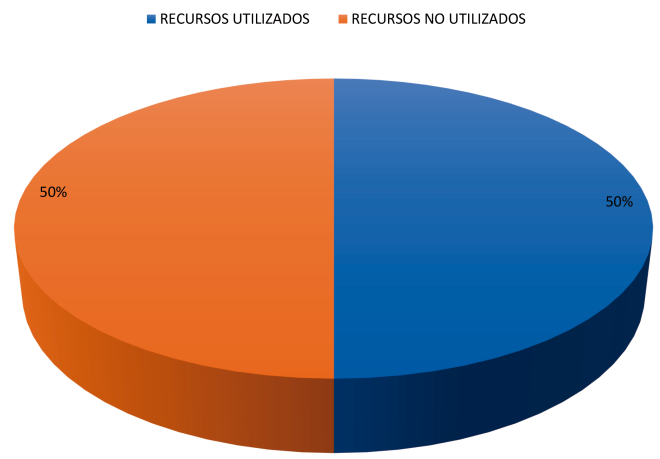

Figura 6. Porcentaje de recursos utilizados en el aula virtual

Es importante mencionar que los docentes utilizan estos recursos en la plataforma virtual, estableciendo que resultan ser las más comunes y de fácil uso para su docencia. (figura 6)

Una vez finalizado este análisis se puede mencionar que los Docentes de la Carrera de Sicología Clínica, limitan el uso de actividades y recursos de un ambiente de aprendizaje de tipo virtual.

Para ello es necesario que los docentes y estudiantes identifiquen su rol dentro de los ambientes de aprendizaje virtuales, para un mejor desempeño, estos roles se detallan a continuación:

\subsection{Roles del Docente y Estudiante en los Ambientes Educativos Virtuales}

Rol Docente. - el profesor cambia de ser un profesor tradicional y modifica su ambiente de enseñanza enriqueciéndose por el uso de las Tecnologías de la Información y Comunicación, pasa a actuar de guía de sus estudiantes para facilitarles en el uso de herramientas y recursos para elaborar nuevos conocimientos y destrezas basados en tecnologías, es aquí y citando a Jesús Salinas en el año 2008, menciona que todo docente debe de ser capaz de:

1) Guiar a sus alumnos en el uso de bases de la información y conocimiento, con el fin de que sus estudiantes tengan en conocimiento necesario para usar recursos tecnológicos.

2) Potenciar que los alumnos se vuelvan activos en el proceso auto dirigido a la hora de construir su conocimiento en ambientes virtuales de aprendizaje.

3) Asesorar y gestionar el ambiente de aprendizaje. Además, tienen que ser capaces de asesorar a sus estudiantes en la adquisición de nuevas experiencias colaborativas, monitorizando su progreso; proporcionando retroalimentación; y ofrecer oportunidades en la difusión de trabajos (Autónomo, Practico y Colaborativo.

4) Un correcto acceso al trabajo del estudiante, manteniendo filosofías y estrategias planteadas en el proceso enseñanza - aprendizaje.

Todo ello trae cambios para el docente, por lo tanto, deben de estar preparados para este rol, donde los estudian- tes sean partícipes de su propio proceso de aprendizaje aplicando una amplia gama de recursos y herramientas que se encuentran disponibles en el internet, es decir implementar más recursos para obtener mejores resultados.

Los docentes constituyen un elemento principal en todo sistema educativo y resultan ser de suma importancia para un buen funcionamiento, por lo tanto, como objetivo principal, deben tener recursos técnicos y didácticos permitiéndoles plasmar y cubrir las necesidades de un sistema educativo basado en ambientes de aprendizaje virtuales buscando la excelencia y la calidad de la educación.

Rol Estudiante. - La utilización de nuevos ambientes de aprendizaje de tipo virtual, implica nuevas consideraciones para el proceso de enseñanza - aprendizaje, en las que como objetivo principal es el de mantener una participación activa por parte de los estudiantes, creando destrezas y adquiriendo nuevos conocimientos para dar soluciones a las problemáticas del mundo de hoy.

Es por esto, que todo estudiante debe mantener cinco perspectivas básicas para una correcta aplicabilidad de estos nuevos entornos virtuales.

1) Acceso a una amplia gama de recursos digitales, en los que se incluyen bases de datos científicas, bibliotecas virtuales, paquetes multimedia, entre otros.

2) Control activo de recursos, donde el estudiante a más de manejar la información debe ser capaz de crear destrezas para usar herramientas de información de tipo virtual.

3) Participación e interacción de nuevas experiencias basadas en el uso de tecnologías, con el fin de gestionar destrezas basadas en las necesidades de cada estudiante.

4) Acceso a grupos de aprendizaje, en lo cual el estudiante pueda mantener un trabajo colaborativo logrando así su madurez, éxito y satisfacción profesional.

5) Ganar una experiencia a la hora de resolver problemas en su proceso de formación académica.

Es importante conocer que hoy en día el mundo educativo requiere una gran atención y como se mencionó en párrafos anteriores, el rápido creciente en el uso de la tecnología permite aprender de mejor manera.

Si a esto sumamos el uso de los nuevos ambientes de aprendizaje, podemos mencionar que deben existir tres aspectos importantes para su correcto funcionamiento y son: Delimitado, se hace referencia a todos los conceptos antes mencionados de los nuevos ambientes de aprendizaje, roles, características, ventajas, entre otras más., Estructurado, en el sentido de que los contenidos deben encontrarse organizados, fomentando una guía para el proceso de enseñanza y Flexible, permitiendo el desarrollo de nuevos criterios para la administración del micro currículo, donde se debe definir dependiendo de ello las competencias que el estudiante va a adquirir en su formación profesional.

Podemos añadir, que los nuevos ambientes de aprendizaje ayudan a la educación, donde los estudiantes pueden mantener un ritmo diferente en su aprendizaje, y resultan 
más aventajados al tener una amplia gama de recursos a su disposición, mientras aquellos que necesiten un refuerzo, el docente deberá implementar materiales de apoyo en línea para el avance de los mismos.

Usar la tecnología en el campo educativo no es algo nuevo hoy en día, sin embargo, la flexibilidad, eficiencia y aprovechamiento de recursos virtuales que se encuentran en los nuevos ambientes de aprendizaje, ofrecen una mayor calidad tanto a docentes como a los estudiantes.

\section{Conclusiones}

Los docentes de la Carrera de Sicología Clínica de la Universidad Católica de Cuenca y después de analizar mediante el enfoque sistémico de cómo se encuentra el sistema a nivel de sílabo; los docentes deben implementar más actividades y recursos en el proceso de enseñanza; además, el resultado del uso fue insatisfactorio dado que en la plataforma virtual se encuentran más actividades y recursos que permitirán plasmar contenidos de mejor manera, manteniendo el objetivo principal de obtener una excelencia en la calidad educativa, a su vez la plataforma llega a ser subutilizada, es decir, solo realizan una carga de archivos y no dan el correcto tratamiento de la información.

Se puede mencionar que los entornos virtuales de enseñanza aprendizaje (EVEA), ofrecen un nuevo mundo de posibilidades de aprendizaje abierto y flexible, pero tanto los docentes como los estudiantes, necesitan buenas condiciones de trabajo, así como un buen funcionamiento adecuado a la red, buscar una eficacia en las instituciones educativas, coherencia en sus planificaciones, y hábitos en los docentes, ya que solo así se puede garantizar la calidad en la educación.

Como se mencionó en párrafos anteriores los nuevos ambientes de aprendizaje fomentan una educación de calidad. Pero son los docentes quienes hacen que esta llegue a cumplirse.

\section{Referencias Bibliográficas}

Alvarez, P., y Cabrera, H. (2016). La influencia de la información digital en la educación. Cuenca, Ecuador: Edúnica.

Arnold, M., y Osorio, F. (1998). Teoría general de sistemas. Universidad de Chile. España: Alianza Editorial.

Cabero, J. (2003). La galaxia digital y la educación: los nuevos entornos de aprendizaje. España: Grupo Comunicar.

Cardona, G. (2006). Tendencias educativas para el siglo xxi. Edutec.

Escontrela, R. (2003). Bases para reconstruir.

Estrada, L. R. G. (1999). Hacia un modelo de evaluación de la calidad de instituciones de educación superior.

Luhmann, N. (1998). Sistemas sociales: lineamientos para una teoría general. Madrid: Alianza Editorial.

Moreira, M. A., Salvat, B. G., y García-Quismondo, M. . M. (2008). Alfabetizaciones y tecnologías de la información y la comunicación. Madrid: Síntesis.
Salinas, J. (1998). El rol del profesorado universitario ante los cambios de la era digital. IV Congreso de Formación para el Trabajo.

Salinas, J. (2005). Nuevos escenarios de aprendizaje. IV Congreso de Formación para el Trabajo.

von Bertalanffy, L. (1984). Tendencias en la teoría genereal de sistemas. Madrid.

Recibido: 1 de septiembre de 2017

Aceptado: 7 de noviembre de 2017 